\title{
A Time-Constant Calibrated Phase-Locked Loop With a Fast-Locked Time
}

\author{
Sung-Rung Han, Student Member, IEEE, Chi-Nan Chuang, Student Member, IEEE, and \\ Shen-Iuan Liu, Senior Member, IEEE
}

\begin{abstract}
A time-constant calibrated phase-locked loop with a fast-locked time is presented. A variable capacitance multiplier (VCM) is developed to adjust the equivalent capacitance in the loop filter. And a calibration circuit is used to allow the time constant of the loop filter to track with the reference clock. By using the proposed time-constant calibration circuit and the VCM, the fast acquisition time is achieved and the loop capacitance is also multiplied. A prototype has been fabricated in a $0.35-\mu \mathrm{m}$ CMOS process to demonstrate the proposed circuit.
\end{abstract}

Index Terms-Fast locked, phase-locked loop (PLL), time-constant calibration, variable capacitance multiplier (VCM).

\section{INTRODUCTION}

$\mathbf{F}$ OR a typical phase-locked loop (PLL), the jitter performance and the transient behavior mainly depend on the dynamic parameters, i.e., damping factor and natural frequency. Moreover, in order to reduce the external components and avoid the undesired noise coupling, the on-chip loop filter is preferred. However, the process and temperature variations alter the dynamic parameters away from the desired values. To tolerate the process and temperature variations, a technique [1] with digitally switched capacitors has been developed to calibrate the loop filter in a PLL. It may have either a complex switched-capacitor array or limited resolution. In this brief, an analog timeconstant calibration circuit is proposed to precisely control the time constant of the loop filter. In order to adjust the equivalent capacitance in the loop filter, a variable capacitance multiplier (VCM) is also developed. To save the power, the clock generator will be stopped and be re-started once the demand is active [2]. By using the proposed time-constant calibration circuit and the VCM, a fast acquisition time for a PLL is achieved. Moreover, the loop capacitance is multiplied and the active area is reduced.

This brief is organized as follows. Section II describes the time-constant calibration circuit. The fast-locking acquisition is discussed in Section III. Section IV illustrates the experimental results. The conclusions are given in Section V.

\section{CIRCUIT DESCRIPTION}

A conventional charge pump PLL is shown in Fig. 1. It is composed of a phase detector, a charge pump, a loop filter, a voltage-controlled oscillator, and a divider. The second-order

Manuscript received December 19, 2005; revised May 24, 2006. This paper was recommended by Associate Editor G.-Y. Wei.

The authors are with Graduate Institute of Electronics Engineering and Department of Electrical Engineering, National Taiwan University, Taipei, Taiwan 10617, R.O.C. (e-mail: 1si@cc.ee.ntu.edu.tw).

Digital Object Identifier 10.1109/TCSII.2006.883826

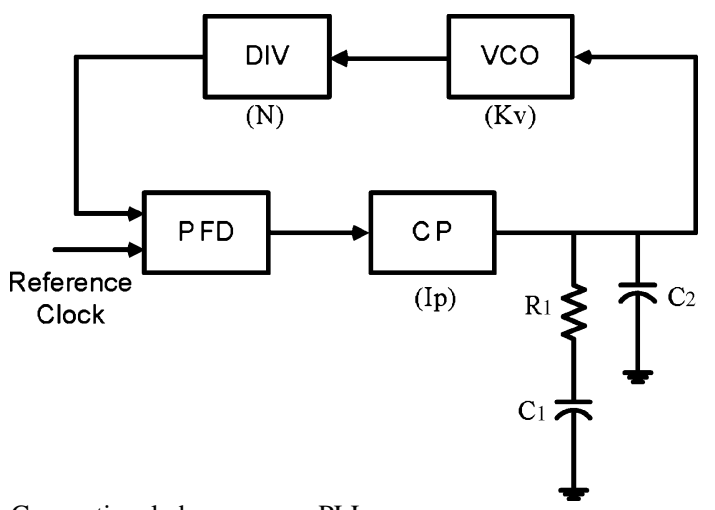

Fig. 1. Conventional charge pump PLL.

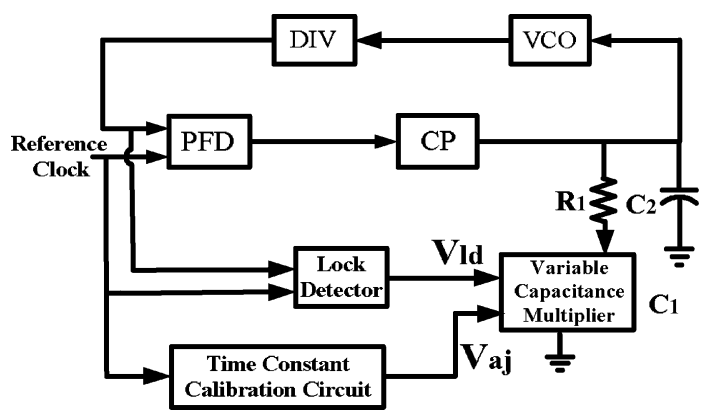

Fig. 2. PLL with a time-constant calibration circuit.

loop filter is composed of the resistor $R_{1}$ and the capacitors $C_{1}$ and $C_{2}$. Assume that $C_{2}$ is much less than $C_{1}$. The damping factor $\zeta$ and natural frequency $\omega_{n}$ are approximately expressed as [3], [4]

$$
\zeta=\frac{R_{1}}{2} \cdot \sqrt{\frac{I_{p} \cdot K_{v} \cdot C_{1}}{N}} \text { and } \omega_{n}=\sqrt{\frac{I_{p} \cdot K_{v}}{N \cdot C_{1}}}
$$

where $N$ is the ratio of the divider, $K_{v}$ is the gain of the VCO, and $I_{p}$ is the charge pump current. Based on the damping factor and the natural frequency, the jitter performance and transient response of a PLL can be estimated. Since the passive components, such as $R_{1}$ and $C_{1}$, vary owing to the process and temperature variations, the damping factor and the natural frequency deviate from the designed values, too. To overcome this problem, an analog time-constant calibration circuit for a PLL is developed.

Assume that the divider's ratio $N$ and the gain of the VCO $K_{v}$ in a PLL are known. Let the charge pump current $I_{p}$ be inversely proportional to $R_{1}$. According to (1), the damping factor and the natural frequency are proportional to $\sqrt{R_{1} \cdot C_{1}}$ and $1 / \sqrt{R_{1} \cdot C_{1}}$, respectively. Fig. 2 presents a PLL with a time-constant calibration circuit, a VCM, and a lock detector. 


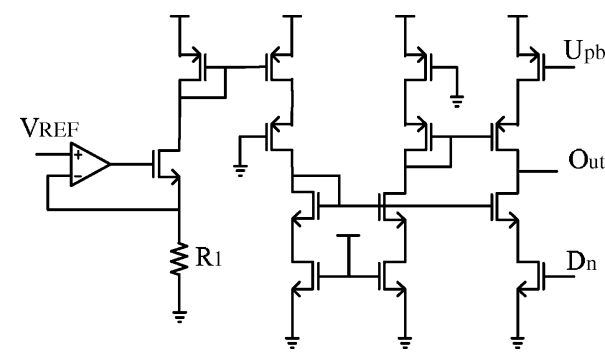

Fig. 3. Charge pump circuit.

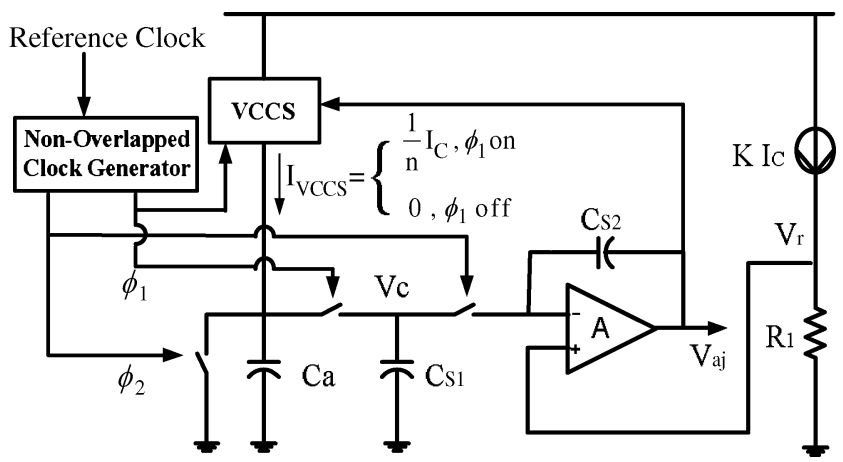

Fig. 4. Time-constant calibration circuit.

The time-constant calibration circuit adjusts the VCM to track the time constant, $R_{1} C_{1}$, of the loop filter with the period $T$ of the reference clock. Thus, the damping factor and natural frequency also track with the period of the reference clock. The detailed circuit descriptions are discussed below.

\section{A. Charge Pump Circuit}

The charge pump circuit is shown in Fig. 3. A reference voltage and a replica resistor, which is equal to the resistor in the loop filter, are connected to the inputs of the amplifier, respectively. Therefore, the charge pump current is inversely proportional to the resistor in the loop filter. This charge pump current is designed around $50 \mu \mathrm{A}$.

\section{B. Time-Constant Calibration Circuit}

Fig. 4 shows the proposed analog time-constant calibration circuit which precisely control the time constant of the loop filter. The replica resistor $R_{1}$ and the replica capacitor $C_{a}$ are equal to the resistor in the loop filter and the capacitor in the VCM, respectively. Two nonoverlapped clocks $\phi_{1}$ and $\phi_{2}$ and the capacitor $C_{a}$ are used to realize an equivalent resistor [5]. The capacitors $C_{s 1}$ and $C_{s 2}$ in the switched-capacitor amplifier are used to sample and integrate the input voltage, respectively. To operate correctly, the capacitor $C_{a}$ must be much larger than $C_{s 1}$. The output current of the voltage-controlled current source (VCCS), $I_{\mathrm{VCCS}}$ is expressed as

$$
I_{\mathrm{VCCS}}= \begin{cases}\frac{I_{C}}{n}, & \phi_{1} \text { on } \\ 0, & \phi_{1} \text { off. }\end{cases}
$$

By the VCCS, the inverting input voltage of the amplifier, $V_{c}$, is approximated to $\left(I_{c} \cdot T\right) /\left(2 \cdot n \cdot C_{a}\right)$ and the noninverting input
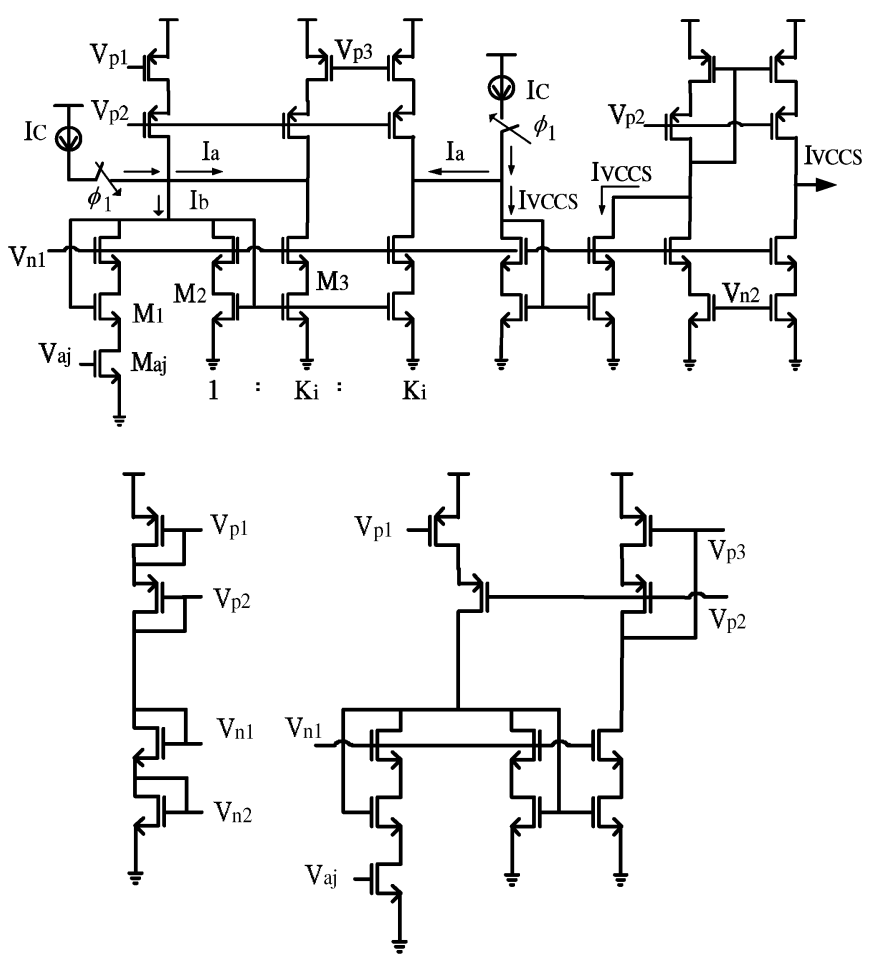

Fig. 5. (a) VCCS. (b) Bias circuits of the VCCS.

voltage of the amplifier $V_{r}$ is equal to $K \cdot I_{c} \cdot R_{1}$ where $K$ is a constant. If there is a difference between these two voltages, the output voltage of the amplifier $V_{a j}$ is altered to adjust the multiplying factor $n$ in the VCCS and the VCM. The effective capacitance $C_{1}$ of the $\mathrm{VCM}$ is designed to be equal to $n C_{a}$. As this circuit reaches its steady state, $V_{c}$ is equal to $V_{r}$ and the time constant $R_{1} C_{1}$ of the loop filter is guaranteed as $T /(2$. $K)$. Thus, the time constant will track with the period of the reference clock.

\section{VCCS}

The VCCS used in the time-constant calibration circuit is shown in Fig. 5(a) and its bias circuits are shown in Fig. 5(b).

In Fig. 5(a), consider only the ac currents. When $\phi_{1}$ is on, the multiplying factor is defined as $n=I_{C} / I_{b}$ and it is variable as in (2). Thus, the ac currents $I_{a}=(1-(1 / n)) I_{C}$ and $I_{\mathrm{VCCS}}=I_{C} / n$ are generated. In Fig. 5(a), the transistor $\mathrm{M}_{a j}$ is biased in the triode region to act as a voltage-controlled resistor. $g_{m 1}, g_{m 2}, R_{a j}$, and $K_{i}$ are the transconductances of M1, $\mathrm{M} 2$, the effective resistance of $\mathrm{M}_{a j}$, and the transistor ratio between M3 and M2, respectively. The ac current $I_{b}$ multiplied by the equivalent resistance $\left(\left(1 / g_{m 1}\right)+R_{a j}\right) / /\left(1 / g_{m 2}\right)$, the transconductance $g_{m 2}$ and the factor $K_{i}$ should be equal to the ac current $I_{a}$. The multiplying factor is derived as

$$
n=1+\frac{K_{i} \cdot g_{m 2} \cdot\left(1+g_{m 1} \cdot R_{a j}\right)}{g_{m 1}+g_{m 2}+g_{m 1} \cdot g_{m 2} \cdot R_{a j}} .
$$

Since $R_{a j}$ is controlled by the voltage $V_{a j}$ from the time-constant calibration circuit, the multiplying factor $n$ is also variable. The nominal value of $n$ is realized around 10 . 


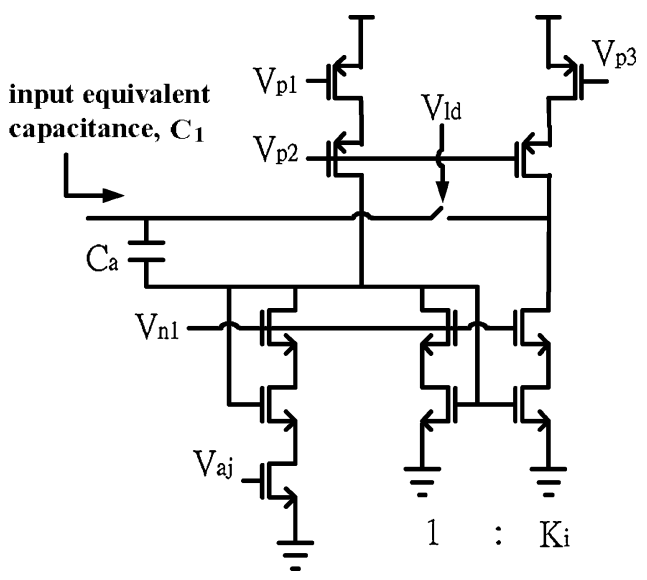

Fig. 6. VCM.

\section{D. $V C M$}

The VCM is a voltage-controlled capacitor as shown in Fig. 6. The capacitance multiplication technique is similar to [6], but our VCM is voltage controlled. It acts as the serial capacitor $C_{1}$ in the loop filter of Fig. 1. When the lock detector [7] is active (i.e., $V_{l d}$ is on), a portion of the input current is bypassed to reduce the current flowing into the capacitor, $C_{a}$. Similar to the VCCS, it results in the effective capacitor $C_{1}$ equal to $n C_{a}$. Also, the voltage $V_{a j}$ adjusts this factor $n$, and therefore, the effective capacitance of $C_{1}=n C_{a}$ in the loop filter is also adjusted. When the lock detector is off (i.e., $V_{l d}$ is off), the effective capacitor $C_{1}=C_{a}$ in the loop filter is not multiplied. The capacitor $C_{a}$ is designed as $15 \mathrm{pF}$ and the effective capacitor $C_{1}$ is varied within $120-175 \mathrm{pF}$.

The multiplying factor $n$ is affected by the supply noise. However, if $g_{m 1}, g_{m 2}$, and $R_{a j}$ are chosen carefully, this issue is greatly reduced. Practically, $K_{v}$ may vary owing to the process and supply variations. The simulated variation of $K_{v}$ is around $\pm 50 \%$. The simulated variation of the damping factor and natural frequency is around $+/-30 \%$ if the time constant is fixed. To cover the full range of the process variations, the calibration range of this method should be increased. Moreover, this method will save the chip area, compared with a conventional PLL using an on-chip passive loop filter.

\section{FAST-LOCKING ACQUISITION}

In general, a PLL is designed with the overdamped transient characteristic to obtain a flat jitter transfer function without peaking. However, in this situation, a long transient time is needed in the acquisition. Thus, if a wide loop bandwidth is used in the transient response, the acquisition time is greatly reduced. To have a low jitter, a narrow-loop bandwidth is desired once the loop is locked. The implementation of this method is discussed as follows. When the lock detector is off, an effective capacitor, $C_{1}=C_{a}$, (i.e., $V_{l d}$ is off) is realized in the loop filter. It equivalently enlarges the loop bandwidth of the PLL. Thus, the fast-locking acquisition is achieved in the transient response. When the lock detector is on, the effective capacitor, $C_{1}=n C_{a}$, is multiplied. It results in a small loop bandwidth and the low jitter performance is obtained. In this design, the capacitor $C_{2}$ is fixed. To avoid the instability issue,
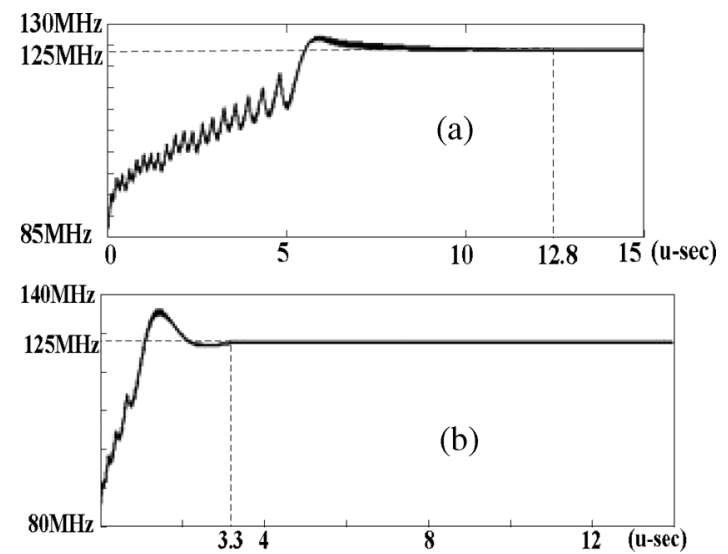

Fig. 7. (a) Simulated transient of the conventional PLL (b) Simulated transient of the proposed PLL.

TABLE I

PERFORMANCE SUMMARY

\begin{tabular}{|l|l|}
\hline Technology & $0.35 \mathrm{um}$ CMOS \\
\hline Supply voltage & $3.3 \mathrm{~V}$ \\
\hline Reference clock & $25 \mathrm{MHz}$ \\
\hline VCO's frequency range & $\begin{array}{l}88-162 \mathrm{MHz} \text { with } \\
\mathrm{Kv}=30 \mathrm{MHz} / \mathrm{V}\end{array}$ \\
\hline Divider & 5 \\
\hline Charge pump current & $50 \mathrm{uA}$ \\
\hline R1 in loop filter & $15 \mathrm{k}$ \\
\hline$C_{1}\left(=n \cdot C_{a}\right.$ ) in the loop filter & $150 \mathrm{pF}$ \\
\hline$C_{2}$ in the loop filter & $10 \mathrm{pF}$ \\
\hline $\begin{array}{l}\text { Calibration range for the time } \\
\text { constant variation }\end{array}$ & $\pm 15 \%$ \\
\hline $\begin{array}{l}\text { Simulated lock time for phase } \\
\text { acquisition (@ 2.25\% UI phase error) }\end{array}$ & $12.8 \mathrm{us}$ (conventional) \\
\hline $\begin{array}{l}\text { Measured locked time } \\
\text { (@ } \pm 2 \% \text { frequency error) }\end{array}$ & $7 \mathrm{us}$ (conventional) \\
\hline Measured rms and peak-to-peak jitters & $2.5 \mathrm{us}$ (proposed) \\
\hline Chip size & $25.4 \mathrm{ps} \mathrm{and} \mathrm{222ps}$ \\
\hline Power consumption & $1.7 \mathrm{mmx} 1.7 \mathrm{~mm}$ \\
\hline
\end{tabular}

the lock window (i.e., the threshold phase difference) of the lock detector is designed to be wide enough.

Fig. 7(a) and (b) shows simulated transient responses for the conventional PLL and the proposed one, respectively. The circuit parameters are listed in Table I. In Fig. 7(a), the locked time is 12.8 us for the phase error less than $2.25 \%$ reference clock period in the conventional PLL. Under the same error criterion, the locked time for the proposed PLL is reduced to be $3.3 \mu \mathrm{s}$.

\section{EXPERIMENTAL RESULTS}

To demonstrate the proposed PLL, a prototype has been fabricated in a $0.35-\mu \mathrm{m}$ CMOS technology. Fig. 8 shows the die photograph. The total area is $1.7 \times 1.7 \mathrm{~mm}^{2}$ with pads and on-chip loop filter. The reference clock is $25 \mathrm{MHz}$. The operation rang of this VCO is $88-162 \mathrm{MHz}$, and the measured $K_{v}$ is $30 \mathrm{MHz} / \mathrm{V}$. The supply voltage is $3.3 \mathrm{~V}$ and the power consumption is $200 \mathrm{~mW}$ including $\mathrm{I} / \mathrm{O}$ buffers.

The measured rms jitter and peak-to-peak one of the proposed PLL in Fig. 9 is 25.4 and 222 ps, respectively. In Fig. 10, the resistor in the loop filter is manually varied with $0.85 R_{1}, R_{1}$, and 


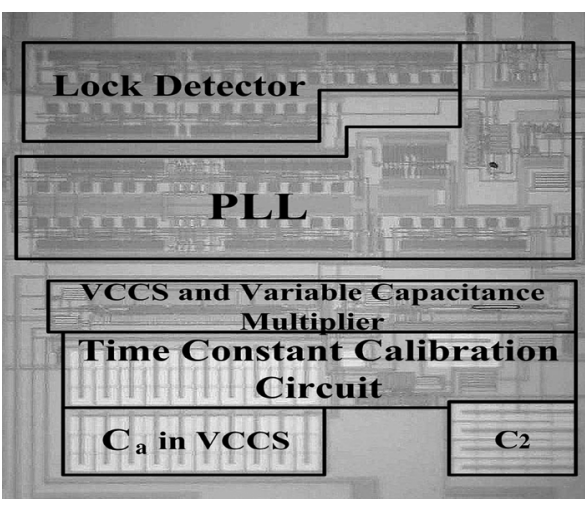

Fig. 8. Die photograph.

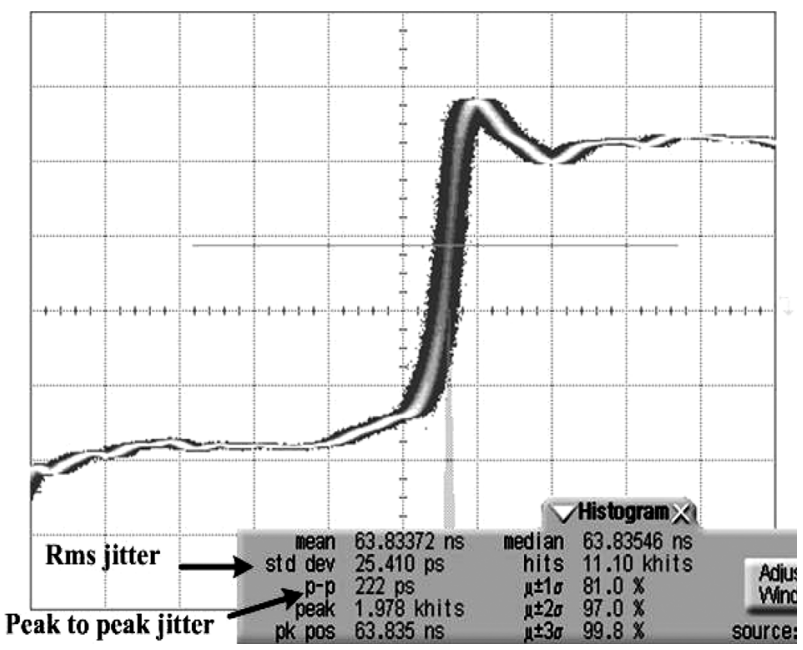

Fig. 9. Measured jitter histogram.

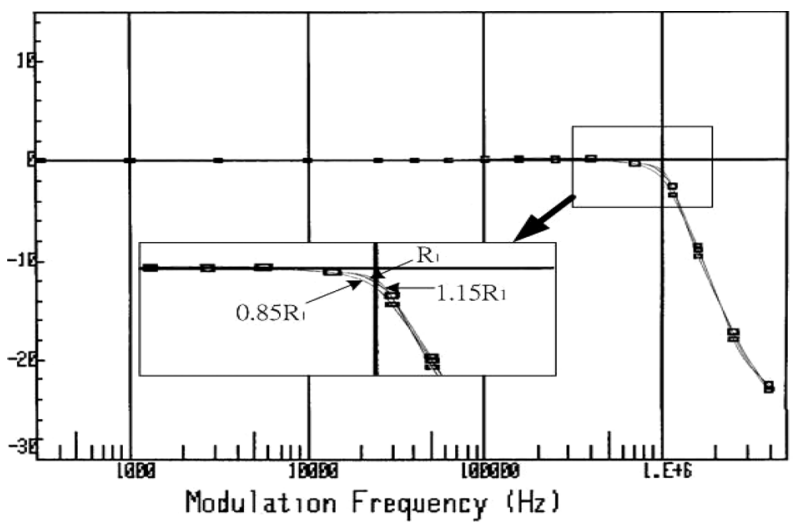

Fig. 10. Measured jitter transfer curves corresponding to different resistances in the loop filter.

$1.15 R_{1}$, respectively. The measured jitter transfer curves corresponding to these resistances are almost the same. It demonstrates that the time constant of the loop filter is calibrated.

Fig. 11 shows the measured transient responses for the conventional PLL and the proposed one to demonstrate the frequency acquisition. The measured locked time within $\pm 2 \%$ frequency error for the conventional PLL and the proposed one is 7 us and $2.5 \mu$ s, respectively. Performance summaries of this work are also listed in Table I.

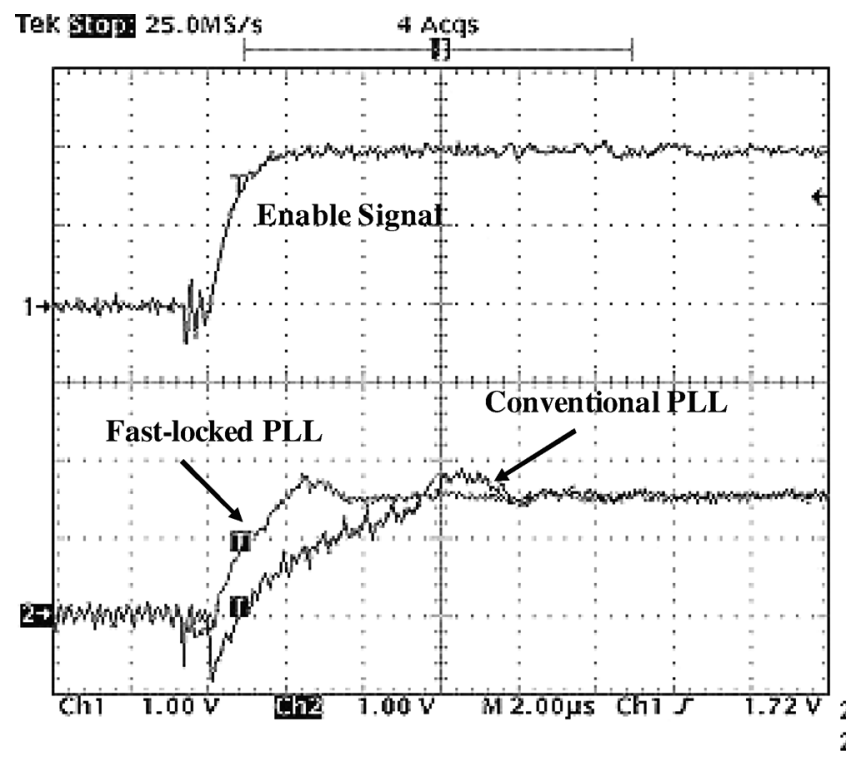

Fig. 11. Measured transient response for the conventional PLL and the proposed one.

\section{CONCLUSION}

In this brief, a time-constant calibrated PLL with a fast-locked time is presented. By using the time-constant calibration circuit and the VCM, the time constant of the loop filter and the dynamic parameters of the PLL track with the period of the reference clock. Moreover, a fast locking acquisition is also achieved and the loop capacitance is multiplied. The measurement results demonstrate the proposed circuits.

\section{ACKNOWLEDGMENT}

The authors would like to thank National Chip Implementation Center (CIC), Taiwan, R.O.C., for fabricating this chip.

\section{REFERENCES}

[1] H. Darabi, S. Khorram, H. M. Chien, M. A. Pan, S. Wu, S. Moloudi, J. C. Leete, J. J. Rael, M. Syed, R. Lee, B. Ibrahim, M. Rofougaran, and A. Rofougaran, "A 2.4-GHz CMOS transceiver for Bluetooth," IEEE J. Solid-State Circuits, vol. 36, no. 12, pp. 2016-2024, Dec. 2001.

[2] Y. Saisaki, N. Kato, and H. Nakaya, "Constant-ratio-coupled multigrain digital synchronizer with flexible input-output delay selection for versatility in low-power applications," IEEE J. Solid-State Circuits, vol. 41, no. 5, pp. 1092-1099, May 2006.

[3] J. Kim, M. Horowitz, and G. Y. Wei, "Design of CMOS adaptive-bandwidth PLL/DLLs: a general approach," IEEE Trans. Circuits Syst. II, Analog Digit. Signal Process., vol. 50, no. 11, pp. 860-869, Nov. 2003.

[4] Z. Wang, "An analysis of charge-pump phase-locked loops," IEEE Trans. Circuits Syst. I, Reg. Papers, vol. 52, no. 10, pp. 2128-2138, Oct. 2005.

[5] P. Roo, S. Sutardja, S. Wei, F. Aram, and Y. Cheng, "A CMOS transceiver analog front-end for Gigabit Ethernet over CAT-5 cables," in IEEE Int. Solid-State Circuits Conf. Dig. Tech. Papers, Feb. 2001, pp. 310-311.

[6] K. Shu, S. S. Edgar, S. M. Jose, and S. H. K. Embabi, "A 2.4-GHz monolithic fractional-N frequency synthesizer with robust phase-switching prescaler and loop capacitance multiplier," IEEE J. Solid-State Circuits, vol. 38, no. 6, pp. 866-873, Jun. 2003.

[7] G. K. Dehng, J. W. Lin, and S. I. Liu, "A fast-lock mixed -mode DLL using a 2-b SAR algorithm," IEEE J. Solid-State Circuits, vol. 36, no. 10, pp. 1464-1471, Oct. 2001. 